\title{
Relevance of 123 I-BMIPP delayed scintigraphic imaging for patients with angina pectoris - a pilot study
}

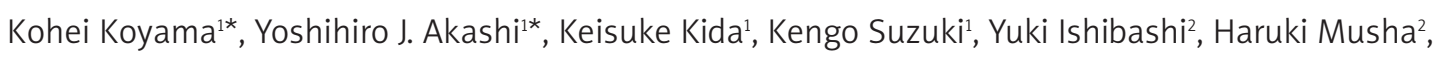
Maciej Banach ${ }^{3}$

${ }^{1}$ Division of Cardiology, Department of Internal Medicine, St. Marianna University School of Medicine, Kawasaki, Japan

2Department of Cardiology, St. Marianna University Yokohama-city Seibu Hospital, Yokohama, Japan

${ }^{3}$ Department of Hypertension, Medical University of Lodz, Lodz, Poland

${ }^{*}$ Both authors contributed equally to this study.

Submitted: 19 April 2011

Accepted: 30 May 2011

Arch Med Sci 2011; 7, 3: 428-432

DOI: 10.5114 /aoms.2011.23407

Copyright (c) 2011 Termedia \& Banach

\section{Abstract}

Introduction: The study was designed to clarify the role of ${ }^{123} \mathrm{I}-\beta$-methyliodophenylpentadecanoic acid (123-BMIPP) in the evaluation of myocardial fatty acid metabolism in patients with stable angina pectoris (AP) before and after percutaneous coronary intervention ( $\mathrm{PCl}$ ).

Material and methods: Ten controls (mean age: $70.4 \pm 10.5$ years) and 12 patients with AP (mean age: $67.4 \pm 11.6$ years) and single vessel coronary artery disease participated in the radionuclide cardiac study. Scintigraphic images were acquired at 30 min and at $4 \mathrm{~h}$ after ${ }^{123}$ I-BMIPP injection to determine early and delayed BMIPP uptake, respectively. The heart-to-mediastinum $(\mathrm{H} / \mathrm{M})$ ratio and the washout rate (WR) were calculated from the planar images. All patients underwent scintigraphy one day before $\mathrm{PCI}$ and again 1 month after successful PCl.

Results: No significant differences in the early or delayed $\mathrm{H} / \mathrm{M}$ ratios were observed between the patients and the controls before PCI (early: $2.70 \pm 0.36$ vs. $2.73 \pm 0.57$; delayed: $2.26 \pm 0.33$ vs. $2.40 \pm 0.43$; $p>0.2$ for both). The early and delayed $\mathrm{H} / \mathrm{M}$ ratios remained unchanged with the comparison with before PCI (early: $2.72 \pm 0.27$, delayed: $2.23 \pm 0.22 ; p>0.2$ for both). The global WR before $\mathrm{PCl}$ was significantly higher in the patients than in the control group $(36.7 \pm 9.3 \%$, vs. $28.1 \pm 8.2 \%, p=0.02$ ). However, the WR after $\mathrm{PCl}$ did not significantly differ between the patients and the controls ( $34.3 \pm 7.8 \%$ vs. $28.1 \pm 8.2 \%, p=0.1)$.

Conclusions: These data may suggest that the WR of ${ }^{123}$ I-BMIPP determined from the planar images enhances the presence of myocardial ischaemia.

Key words: angina pectoris, $\beta$-methyl-iodophenylpentadecanoic acid, delayed image, emission computed tomography.

\section{Introduction}

Myocardial fatty acid metabolism is widely assessed using radioiodinelabelled 15-(p-iodophenyl)-3-(R,S)-methyl pentadecanoic acid (I-BMIPP) $[1,2]$. The clinical use of I-BMIPP has been approved worldwide. Ischaemic memory can be investigated by fatty acid imaging using single photon emission computed tomography (SPECT) with radioiodine-labelled fatty acid analogues such as I-BMIPP [3]. Fatty acid metabolism may be

\author{
Corresponding author: \\ Yoshihiro J. Akashi MD, PhD \\ Division of Cardiology \\ Department of Internal \\ Medicine \\ St. Marianna University \\ School of Medicine \\ 2-16-1 Sugao Miyamae-ku \\ Kawasaki-city \\ Kanagawa-prefecture \\ 216-8511 Japan \\ Phone: +81 449778111 \\ Fax: +81 449767093 \\ E-mail: \\ yoakashi-circ@umin.ac.jp
}


suppressed for a prolonged period after an ischaemic episode and I-BMIPP imaging can visualize regional metabolic defects [4]. Basic kinetics and clinical implications have also been extensively investigated using I-BMIPP [5-9]. Thus, SPECT using I-BMIPP is clinically useful to diagnose myocardial ischaemia and to assess the prognosis of previous myocardial infarction [6] even in patients with angina pectoris (AP) $[7,10]$. A recent study has identified I-BMIPP as a useful tracer for defining regional ischaemic memory in patients with exercise-induced myocardial ischaemia [3]. Our previous study also suggested that ${ }^{123} \mid-\beta$-methyliodophenylpentadecanoic acid (123I-BMIPP) myocardial scintigraphy is a useful tool to predict masked myocardial disease [8]. However, no studies have fully investigated the effectiveness of radioisotopic ${ }^{123}$ I-BMIPP in the evaluation of myocardial fatty acid metabolism in patients with stable AP after percutaneous coronary intervention $(\mathrm{PCI})$. The present study aims to clarify the value of determining fatty acid metabolism using ${ }^{123}$-BMIPP in such patients before and after $\mathrm{PCl}$.

\section{Material and methods}

\section{Subjects}

This study included 12 patients with AP (mean age of $67.4 \pm 11.6$ years) who underwent myocardial ${ }^{123}$-BMIPP imaging and 10 healthy controls (mean age of $70.0 \pm 9.5$ years). Coronary angiography confirmed AP in the 12 patients, all of whom had single vessel coronary artery disease. Of these, eight had AP in the left descending coronary artery, and two each had AP in the left circumflex and right coronary arteries respectively. The mean score of disease severity was $2.1 \pm 0.7$ according to the Canadian Cardiovascular Society (CCS) classification [11]. None of the patients had unstable AP, and all of them met the diagnostic criteria for ischaemia in initial exercise stress testing. None had a history of myocardial infarction or coronary intervention. All patients were prescribed with anti-platelet medication and seven of them were also under statin therapy to control dyslipidaemia.

The healthy controls underwent symptomlimited exercise stress testing to confirm that they were free of ischaemic heart disease. All controls achieved their target heart rates in sinus rhythm without ischaemic ST-T changes or any complaints. None of them had a history of cardiac disease or any other extant medical conditions except hyperlipidaemia. Cardiac function was assessed in the controls by two-dimensional echocardiography (SONOS-2500, Hewlett-Packard Co., MA, USA) to exclude the possibility of having major heart disease before the stress test.

\section{Blood examination}

Venous blood $(5 \mathrm{ml})$ was collected from the antecubital vein of each participant immediately before the scintigraphic study to measure levels of plasma brain natriuretic peptide (BNP). The blood samples were immediately cooled on ice in polypropylene tubes containing $4.5 \mathrm{mg}$ of EDTA-2Na, and then plasma samples separated by centrifugation at 3,000 rpm for $10 \mathrm{~min}$ at $4^{\circ} \mathrm{C}$ were stored frozen at $-70^{\circ} \mathrm{C}$. Plasma BNP concentrations were measured using an immunoradiometric assay with two monoclonal antibodies.

\section{3|-BMIPP myocardial scintigraphy}

We acquired SPECT images at 30 min (early) and at $4 \mathrm{~h}$ (delayed) after ${ }^{123}$ I-BMIPP (111MBq; Nihon Medi-physics Co. Ltd., Tokyo, Japan) was injected into the left antecubital vein to determine uptake. The heart-to-mediastinum (H/M) ratio and the washout rate (WR) were calculated from the planar images.

Anterior and lateral planar images were acquired for $90 \mathrm{~s}$ before the SPECT study using a gamma camera with a low-energy, generalpurpose collimator and a $256 \times 256$ matrix. We acquired BMIPP images using a double-head gamma camera (E.CAM duet, Toshiba Medical, Tokyo, Japan) with a low-energy, general-purpose collimator. Two detectors $\left(2 \times 180^{\circ}\right)$ acquired 60 views for $19 \mathrm{~s}$ in $6^{\circ}$ steps using a $64 \times 64$ matrix. The energy window of ${ }^{123}$ I was centred at $160 \mathrm{keV}$ $\pm 10 \%$. Transaxial images were reconstructed after filtered back projection using a Butterworth filter (order, 8; cut-off frequency, 0.27 cycles/cm). Coronal slices were generated after reorienting the axes. Standard electrocardiography gated images were acquired over 64 steps in 19 s per step in the step-acquisition mode in the R-R interval and divided into 16 frames. Tracer uptake was assessed by creating non-gated early images from the sum of all gated images obtained in the standard-acquisition mode.

All patients underwent scintigraphy 1 day before, and again at 1 month after successful PCl. Coronary angiography confirmed successful revascularization at 3 months after $\mathrm{PCl}$. The controls were also assessed by scintigraphy.

\section{Data analysis}

Regions of interest (ROIs) were drawn over the entire heart and upper mediastinum in the planar images. The H/M ratio and global WR of ${ }^{123}$ I-BMIPP were calculated based on the ROIs as follows:

$\mathrm{H} / \mathrm{M}=$ mean pixel count of cardiac ROI/mean pixel count of mediastinal ROI; WR $(\%)=[$ (mean early cardiac pixel count - mean delayed cardiac pixel count)/mean early cardiac pixel count] $\times 100$. 
Table I. Subjects' background

\begin{tabular}{|c|c|c|}
\hline & $A P$ & Control \\
\hline Patients No. & 12 & 10 \\
\hline Age [years] & $67.4 \pm 11.6$ & $70.0 \pm 9.5$ \\
\hline Male/female & $9 / 4$ & $5 / 6$ \\
\hline \multicolumn{3}{|l|}{ Culprit } \\
\hline LAD (cases) & 8 & \\
\hline LCX (cases) & 2 & \\
\hline RCA (cases) & 2 & \\
\hline CCS class & $2.1 \pm 0.7$ & \\
\hline LVEF by QGS (\%) & $64.0 \pm 13.7$ & $70.4 \pm 10.5$ \\
\hline $\mathrm{BNP}[\mathrm{pg} / \mathrm{ml}]$ & $55.1 \pm 21.0$ & $26.5 \pm 15.4$ \\
\hline \multicolumn{3}{|l|}{ Medications } \\
\hline Anti-platelet & 12 & \\
\hline ACE-I & 1 & \\
\hline ARB & 2 & \\
\hline$\beta$-blockade & 4 & \\
\hline Ca-blockade & 3 & \\
\hline Nitroglycerin & 5 & \\
\hline Statin & 7 & 3 \\
\hline
\end{tabular}

$A P$ - angina pectoris, $L A D$ - left anterior descending, $L C X$ - left circumflex, RCA - right coronary artery, CCS - Canadian Cardiovascular Society, LVEF - left ventricular ejection fraction, QGS - quantitive gated SPECT, BNP - brain natriuretic peptide, ACE-I - angiotensin converting enzyme inhibitor, $A R B$ - angiotensin receptor blockade, $\mathrm{Ca}$ - calcium. Some values are expressed as mean $\pm S D$

Background and time decay were not corrected to calculate the WR.

After a SPECT image was acquired and reconstructed, the left ventricular (LV) myocardium was divided into 17 segments as recommended [12] and then segment defects were scored as 0 (normal), 1 (slightly reduced), 2 (mildly reduced), 3 (severely reduced) and 4 (absent). The total defect score (TDS) was defined as the sum of the segment defect scores for each participant.

Ventricular edges and LV ejection fraction (LVEF), LV end-diastolic volume (LVEDV) and LV end-systolic volume (LVESV) were evaluated using quantitative gated SPECT (QGS) software (Cedars-Sinai Medical Center, Los Angeles, CA). The algorithm for determining edges and calculating volume has been described previously [13].

\section{Ethics}

The study protocol consisting of blood tests, echocardiography, an exercise stress test, cardiac catheterization, and a BMIPP radionuclide study proceeded in accordance with the ethical principles established in the Declaration of Helsinki. The Human Investigation Committee of St. Marianna University School of Medicine approved the study protocol. The nature and purpose of the study and involved risks were fully explained to all participants who then provided written informed consent to participate in all procedures associated with the study before enrolment.

\section{Statistical analysis}

Results are expressed as means \pm SD. The significance of differences between the patients with AP and the controls was analysed using an unpaired $t$ test. Differences before and after $\mathrm{PCl}$ were analysed and the parameters of early and delayed data in the same individual were compared using a paired $t$ test. A $p$ value of $<0.05$ was considered statistically significant.

\section{Results}

\section{Baseline characteristics}

Table I shows the clinical characteristics of the patients and controls. Age did not significantly differ between the two groups $(p>0.2)$. The QGS data uncovered no significant differences in LVEF between patients before $\mathrm{PCl}$ and the controls (64.0 $\pm 13.7 \%$ vs. $70.4 \pm 10.5 \%, p>0.2$ ). The plasma BNP concentration significantly differed between patients with AP before PCl and the controls $(55.1 \pm 21.0 \mathrm{pg} / \mathrm{ml}$ vs. $26.5 \pm 15.4 \mathrm{pg} / \mathrm{ml}, p=0.0018$ ). All patients were medicated with $100 \mathrm{mg}$ of aspirin per day. Seven of the patients and 3 controls were prescribed with statins to reduce serum cholesterol concentrations.

\section{BMIPP scintigraphic study}

\section{SPECT images}

The early and delayed TDSs in the controls were $1.2 \pm 0.5$ and $2.1 \pm 0.5$, respectively. Successful $\mathrm{PCl}$ decreased the early TDS from $5.5 \pm 1.8$ to $2.8 \pm 1.1$ in the patients $(p<0.001)$. Moreover, the delayed TDS in the patients significantly decreased from $7.5 \pm 1.7$ to $5.3 \pm 1.8$ after $\mathrm{PCl}(p<0.001)$.

The early TDSs were significantly higher before and after $\mathrm{PCl}$ in the patients than in the controls (before $\mathrm{PCl}, p<0.001$; after $\mathrm{PCl}, p=0.004$ ). The delayed TDSs in the patients before and after $\mathrm{PCl}$ were also higher than delayed TDSs in the controls ( $p<0.001$ for both groups).

There were no significant differences in LVEDV (controls $44.6 \pm 14.5 \mathrm{ml}$; before $\mathrm{PCl} 51.4 \pm 12.3 \mathrm{ml}$; after $\mathrm{PCl} 53.0 \pm 16.0 \mathrm{ml}$ ), LVESV (controls $14.3 \pm 8.6 \mathrm{ml}$; before $\mathrm{PCl} 19.3 \pm 9.2 \mathrm{ml}$; after $\mathrm{PCl} 21.5 \pm 12.1 \mathrm{ml}$ ) or LVEF (controls $70.4 \pm 10.5 \%$; before PCI $64.0 \pm 13.7 \%$; after $\mathrm{PCl} 62.3 \pm 13.4 \%$ ) between the controls and patients before and after $\mathrm{PCl}$ (all $p>0.2$; Figure 1 top).

\section{Planar images}

The early and delayed H/M ratios did not significantly differ between the patients before $\mathrm{PCl}$ 

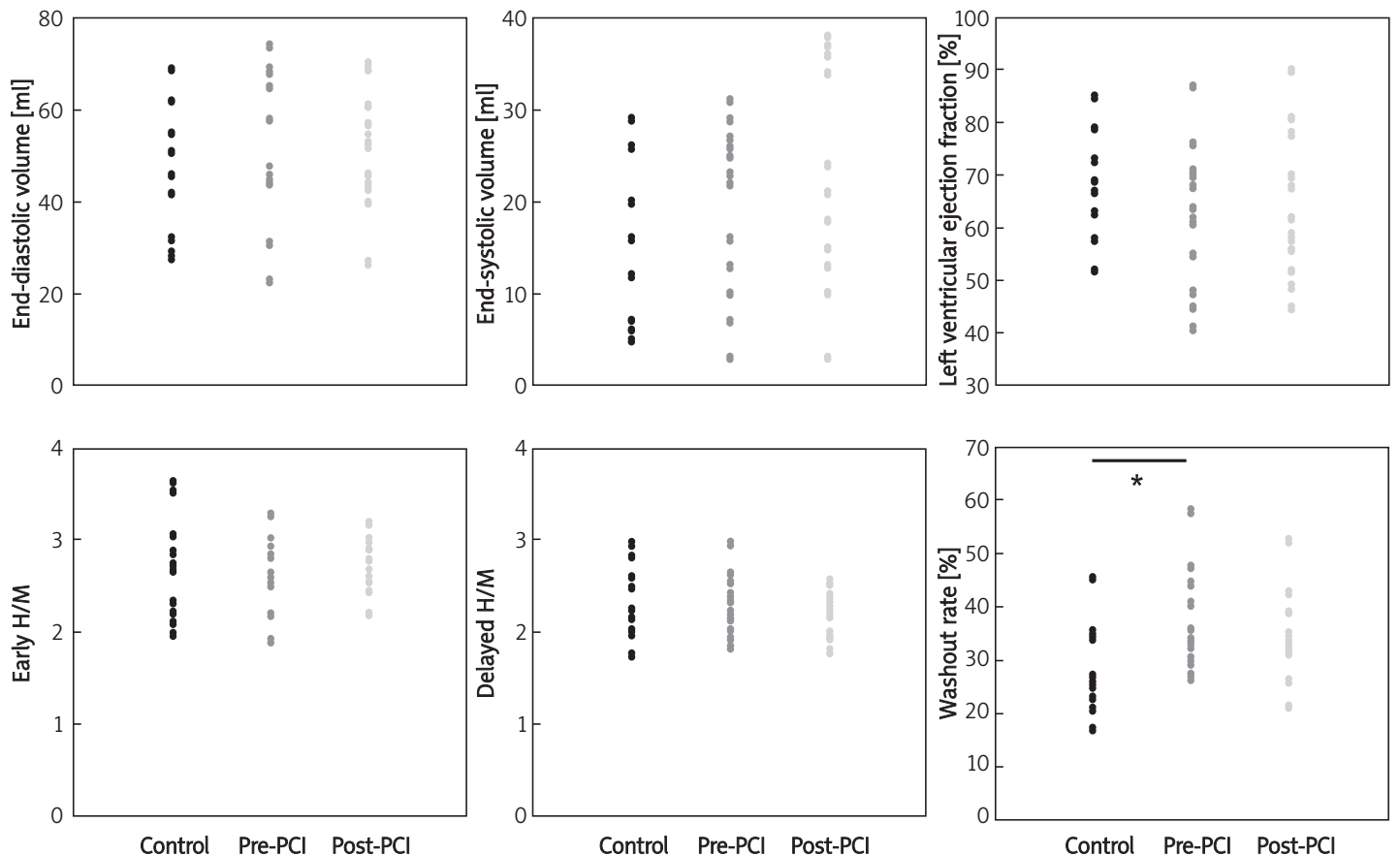

Figure 1. Quantitative gated single photon emission computed tomography findings. Top: Parameters in controls, before and after $\mathrm{PCI}^{*}$. There were no significant differences in LVEDV $(44.6 \pm 14.5 \mathrm{ml}, 51.4 \pm 12.3 \mathrm{ml}$ and $53.0 \pm 16.0 \mathrm{ml}$, respectively), LVESV $(14.3 \pm 8.6 \mathrm{ml}, 19.3 \pm 9.2 \mathrm{ml}$ and $21.5 \pm 12.1 \mathrm{ml}$, respectively) or LVEF $(70.4 \pm 10.5 \%, 64.0 \pm 13.7 \%, 62.3$ $\pm 13.4 \%$, respectively). Bottom: Planar image shows no significant differences in early or delayed $\mathrm{H} / \mathrm{M}$ ratios between patients with AP before $\mathrm{PCl}$ and controls (early $2.70 \pm 0.36$ vs. $2.73 \pm 0.57$; delayed $2.26 \pm 0.33$ vs. $2.40 \pm 0.43$, all $p>0.2$ ). Early and delayed $\mathrm{H} / \mathrm{M}$ ratios after $\mathrm{PCl}$ remained unchanged compared with those before $\mathrm{PCl}$ (early $2.72 \pm 0.27$; delayed $2.23 \pm 0.22$, all $p>0.2$ ). Global WR before $\mathrm{PCl}$ was significantly higher in patients than in controls $(36.7 \pm 9.3 \%$ vs. $28.1 \pm 8.2 \%, p=0.025)$. However, WR after PCI did not significantly differ between the AP patients and the controls $(34.3 \pm 7.8 \%$ vs. $28.1 \pm 8.2 \%, p=0.10)$

${ }^{*}$ All abbreviations are explained in the text of the manuscript

and the controls (early $2.70 \pm 0.36$ vs. $2.73 \pm 0.57$; delayed $2.26 \pm 0.33$ vs. $2.40 \pm 0.43$, all $p>0.2$ ) and remained unchanged after $\mathrm{PCl}$ compared with those before $\mathrm{PCl}$ (early $2.72 \pm 0.27$; delayed 2.23 \pm 0.22 , all $p>0.2$ ). The global WR before $\mathrm{PCI}$ was significantly higher in the patients than in the controls ( $36.7 \pm 9.3 \%$ vs. $28.1 \pm 8.2 \%, p=0.025$ ), whereas the WR after $\mathrm{PCl}$ did not significantly differ between them ( $34.3 \pm 7.8 \%$ vs. $28.1 \pm 8.2 \%, p=0.10$; Figure 1 bottom).

\section{Discussion}

The present study discovered that the global WR of BMIPP in the myocardium was accelerated in patients with stable AP before $\mathrm{PCI}$ but did not differ from control values after successful PCI.

The significance of BMIPP myocardial WR remains a matter of debate. We previously showed that an accelerated BMIPP WR indicated myocardial disease [8]. The WR of BMIPP could not fully discriminate full from partial BMIPP $\beta$-oxidation in the myocardium, although one study demonstrated that BMIPP washout and back-diffusion significantly increase in patients with heart failure [14]. The present study included only patients with stable AP, and the WR of BMIPP might be accelerated even more in patients with unstable AP. The $\beta$-methyl side-branch of BMIPP prolongs BMIPP accumulation in the myocardium through impeding $\beta$-oxidation [1]. Actual myocardial fatty acid disorders might have been reflected more accurately by delayed than by early uptake in the present study, because of the slow myocardial clearance of BMIPP. Mitochondrial fatty acid metabolism might be inhibited in patients with AP and myocardial ischaemia. Thus, the delayed $\mathrm{H} / \mathrm{M}$ ratios did not significantly differ between patients with AP after $\mathrm{PCl}$ and the controls. Our previous study demonstrated lower BMIPP uptake in patients with heart failure than with AP [8]. This is attributed to a myocardial energy deficiency in the failing heart [9].

Stress tests can be restricted in elderly patients with orthopaedic disease and in those with bronchial asthma. The administration of contrast media can induce nephropathy in patients with chronic kidney disease which is often fatal $[15,16]$. However, myocardial ischaemia can be identified by BMIPP image acquisition even in planar images, without the need for stress tests. The non-invasive aspect of BMIPP imaging is important for evaluating the effect of catheter revascularization in patients 
with various cardiac diseases. This method would allow further clinical assessments in patients with various myocardial diseases.

The study population was small and thus further studies on a larger population are required. The regional WR of ${ }^{123}$ I-BMIPP should also be evaluated. Although the prognostic value of the $\mathrm{H} / \mathrm{M}$ ratios and WR of ${ }^{123}$ I-BMIPP in patients with cardiac disease remains unknown, this question should be resolved to define how ${ }^{123}$ |-BMIPP can be optimally applied as a prognostic indicator.

In conclusion, the present findings suggest that the ${ }^{123}$ I-BMIPP WR obtained from planar images enhances the likelihood of myocardial ischaemia. Planar BMIPP images are still useful to determine the outcomes of $\mathrm{PCl}$. Further investigations are needed to reach significant conclusions regarding measurements of delayed $\mathrm{H} / \mathrm{M}$ ratios in patients with ischaemic heart disease.

\section{References}

1. Fujibayashi Y, Yonekura Y, Takemura Y, et al. Myocardial accumulation of iodinated beta-methyl-branched fatty acid analogue, iodine-125-15-(p-iodophenyl)-3-(R,S)methylpentadecanoic acid (BMIPP), in relation to ATP concentration. J Nucl Med 1990; 31: 1818-22.

2. Fujibayashi Y, Nohara R, Hosokawa R, et al. Metabolism and kinetics of iodine-123-BMIPP in canine myocardium. J Nucl Med 1996; 37: 757-61.

3. Dilsizian V, Bateman TM, Bergmann SR, et al. Metabolic imaging with beta-methyl-p-[(123)I]-iodophenyl-pentadecanoic acid identifies ischemic memory after demand ischemia. Circulation 2005; 112: 2169-74.

4. Tamaki N, Tadamura E, Kawamoto M, et al. Decreased uptake of iodinated branched fatty acid analog indicates metabolic alterations in ischemic myocardium. J Nucl Med 1995; 36: 1974-80.

5. Nohara R, Hosokawa R, Hirai T, et al. Basic kinetics of $15-$ (p-iodophenyl)-3-R,S-methylpentadecanoic acid (BMIPP) in canine myocardium. Int J Card Imaging 1999; 15: 11-20.

6. Matsuki T, Tamaki N, Nakata T, et al. Prognostic value of fatty acid imaging in patients with angina pectoris without prior myocardial infarction: comparison with stress thallium imaging. Eur J Nucl Med Mol Imaging 2004; 31: 1585-91.

7. Chikamori T, Fujita H, Nanasato M, Toba M, Nishimura T. Prognostic value of I-123 15-(p-iodophenyl)-3-(R,S) methylpentadecanoic acid myocardial imaging in patients with known or suspected coronary artery disease. J Nucl Cardiol 2005; 12: 172-8.

8. Akashi YJ, Kida K, Suzuki K, et al. The significance of 123IBMIPP delayed scintigraphic imaging in cardiac patients. Int J Cardiol 2007; 117: 145-51.

9. Kida K, Akashi YJ, Yoneyama K, Shimokawa M, Musha H. 123I-BMIPP delayed scintigraphic imaging in patients with chronic heart failure. Ann Nucl Med 2008; 22: 769-75.

10. Nakata T, Kobayashi T, Tamaki N, et al. Prognostic value of impaired myocardial fatty acid uptake in patients with acute myocardial infarction. Nucl Med Commun 2000; 21: 897-906.

11. Campeau L. Letter: Grading of angina pectoris. Circulation 1976; 54: 522-3.

12. Cerqueira MD, Weissman NJ, Dilsizian V, et al. Standardized myocardial segmentation and nomenclature for tomographic imaging of the heart: a statement for healthcare professionals from the Cardiac Imaging Committee of the Council on Clinical Cardiology of the American Heart Association. Circulation 2002; 105: 539-42.

13. Germano G, Kiat H, Kavanagh PB, et al. Automatic quantification of ejection fraction from gated myocardial perfusion SPECT. J Nucl Med 1995; 36: 2138-47.

14. Kataoka K, Nohara R, Hosokawa R, et al. Myocardial lipid metabolism in compensated and advanced stages of heart failure: evaluation by canine pacing model with BMIPP. J Nucl Med 2001; 42: 124-9.

15. Best PJ, Lennon R, Ting HH, et al. The impact of renal insufficiency on clinical outcomes in patients undergoing percutaneous coronary interventions. J Am Coll Cardiol 2002; 39: 1113-9.

16. Amin $\mathrm{H}$, Aronow WS, Lleva P, et al. Prevalence of transthoracic echocardiographic abnormalities in patients with ischemic stroke, intracerebral hemorrhage, and subarachnoid hemorrhage. Arch Med Sci 2010; 6: 40-2. 